\title{
Previsão de cargas elétricas através do backpropagation estocástico
}

\author{
Camilla N. S. Mota ${ }^{1}$ \\ UNESP, Ilha Solteira, SP \\ Mara L. M. Lopes ${ }^{2}$ \\ UNESP, Ilha Solteira, SP
}

\begin{abstract}
Resumo.A previsão de cargas elétricas vem se tornando uma atividade imprescindível para o funcionamento e operação dos sistemas elétricos de potência, tendo estes como objetivo fornecer energia elétrica aos consumidores de maneira segura, eficaz e com menor custo de geração possível. O modelo proposto do sistema previsor de cargas elétricas é de natureza computacional, fundamentado em uma rede neural Perceptron, via algoritmo backpropagation estocástico, visando a convergência da rede neural e escapando dos mínimos locais que são problemas apresentados pelo algoritmo backpropagation convencional. Os resultados obtidos na comparação se baseiam no mesmo banco de dados históricos de uma companhia de setor elétrico brasileiro.
\end{abstract}

Palavras-chave. Redes Neurais Artificiais, Backpropagation, Processo Estocástico, Previsão de Cargas Elétricas.

\section{Introdução}

Os métodos relacionados à previsão de cargas elétricas encontrados na literatura, utilizam com muita frequência os modelos matemáticos como: alisamento exponencial, técnica de regressão linear, filtro de Kalman, ARIMA de Box e Jenkins [8]. Esses mecanismos citados têm conseguido, com maior ou menor exatidão, executar a previsão de carga. O sistema é influenciado por fatores externos e a não-linearidade, tendo em vista que podem interferir na modelagem da carga tornando a sua modelagem mais complexa. Dentre estes fatores tem-se: condições meteorológicas, hábitos de consumo, dias de semana, e dias atípicos,também denominadas de variáveis exógeneas [1]. Devido às grandes quantidades de dados de cargas e a não-linearidade, pesquisadores estudam técnicas de inteligência artificial para propor uma solução para suprir as dificuldades dos métodos matemáticos já existentes. Entre essas técnicas destacam-se a Lógica Fuzzy, redes neurais, sistemas especialistas, etc.

As Redes Neurais Artificiais, têm se apresentado como um dos procedimentos mais eficientes para a obtenção da previsão de cargas elétricas, as quais são modelos computacionais inspirados no sistema nervoso de seres vivos, possuindo capacidade de aquisição e manutenção do conhecimento baseado em informações. A modelagem matemática é inspirada no neurônio artificial. Diversos pesquisadores continuaram o trabalho de desenvolvimento através desse modelo, porém com resultados poucos significativos, passando por um período em que pouquíssimas pesquisas eram desenvolvidas. Somente após os anos 70, com a proposição por Hopfield (1982) [9] de redes recorrentes baseadas em funções de energia, fez com que as áreas de redes neurais artificiais retomassem o destaque. A retomada definitiva das pesquisas se deve a diversos fatores, tais como o

\footnotetext{
${ }^{1}$ camilla.mota@unesp.br

2 mara.lopes@unesp.br
} 
desenvolvimento de computadores com maior capacidade de armazenamento, criação de algoritmos de otimização, destacando o algoritmo denominado backpropagation [10], que foi desenvolvido independentemente por pesquisadores em três ocasiões: 1974,1982 e 1986.

O algoritmo em destaque backpropagation, trata-se de um treinamento supervisionado, o qual considera o conjunto de padrões de entrada, que fornecem as saídas desejadas através do ajuste de pesos da rede neural, considerado um dos melhores em relação à precisão, porém existem algumas limitações como velocidade e qualidade de convergência. Em vista dessa deficiência, este artigo tem como objetivo desenvolver uma metodologia para previsão de cargas elétricas, utilizando como ferramenta o algoritmo backpropagation estocástico. Pretende-se a melhoria do treinamento, na sua convergência e escapando dos mínimos locais, tornando o sistema previsor mais eficiente e competitivo em relação aos métodos convencionais.

\section{Backpropagation Estocástico aplicado na Previsão de Carga Elétrica}

O previsor de cargas elétricas proposto neste artigo é fundamentado em uma rede neural Perceptron, via algoritmo backpropagation estocástico. Tem-se uma técnica de atualização de pesos da rede baseados no método estocástico, no qual nem todos os pesos são atualizados de maneira ordenada, tendo este como objetivo uma melhor convergência da rede e a confiabilidade dos seus resultados. O desenvolvimento dessa metodologia, baseia-se nos seguintes fundamentos:

\subsection{Previsão de cargas}

A previsão de cargas elétricas é um requisito fundamental para o setor energético, uma vez que baseado nos seus resultados, decisões importantes são apanhadas. Esta análise é essencial para a área de planejamento da expansão, fluxo de potência, estabilidade transitória, análise, controle de Segurança do Sistema de Energia Elétrica [5].

A metodologia proposta, do sistema previsor de cargas elétricas, tem por finalidade estabelecer previsões com melhores precisões frente a fatores externos e a não-linearidade presente no modelo. Para execução do modelo é importante que ocorram duas condições fundamentais [8]: a primeira refere-se ao foto que as informações ocorridas no passado devem ser quantificadas em forma de dados e a segunda é assumir que o comportamento ocorrido no passado de certa forma irá se repetir no futuro.

A modelagem de cargas elétricas trata-se de um sistema não-linear e não apresentam resultados apropriados, dessa maneira as novas metodologias como: redes neurais artificiais, lógica nebulosa(fuzzy), sistemas híbridos, entre outros [2], vem trazendo significantes resultados. Neste trabalho será abordada a previsão de cargas elétricas empregando as redes neurais artificiais, com previsão de curto prazo (previsão diárias), para prever 24 horas subsequentes. O comportamento do sistema neural, é realizado através de um processo de treinamento, via algoritmo backpropagation estocástico.

\subsection{Redes neurais artificiais}

As redes neurais artificiais apresentam performances boas ou melhores que as demais técnicas disponíveis para a previsão de cargas elétricas. Portanto, esta técnica torna-se o mecanismo principal para essa previsão por duas razões: o fato das redes neurais poderem aproximar numericamente qualquer função desejada e outro por não dependerem dos demais modelos. 
As estruturas das redes neurais artificias foram desenvolvidas com base no modelo de um neurônio e do cérebro humano. Esses modelos foram inspirados com princípio da análise de geração e propagação de impulsos elétricos pela membrana celular dos neurônios [3].

Os modelos de neurônios artificiais utilizados nas redes neurais são não-lineares, fornecem saídas contínuas, coletam os sinais existentes em suas entradas e agregam de acordo com a função operacional, produzindo uma reposta, levando em consideração a função de ativação [6].

\subsection{Treinamento da rede}

Um dos ressaltos das redes neurais artificiais está na capacidade de aprender. Para tanto deve ser treinada, através de um processo iterativo de ajustes aplicados aos seus pesos. Esse processo de treinamento consiste da aplicação de passos ordenados, o que é denominado de algoritmo de aprendizagem.

Neste artigo será abordado o algoritmo de backpropagation, denominada de "propagação reversa", que é uma técnica que adapta os pesos da rede em direção oposta à direção do gradiente descendente, tendo como objetivo minimizar o erro quadrático da rede neural. A soma do erro quadrático instantâneo de cada neurônio da última camada (saída da rede), é dada pela equação (1) $[11]:$

$$
\epsilon^{2}=\sum_{i=1}^{n s} \epsilon_{i}^{2}
$$

Onde $\epsilon^{2}=d_{i}-y_{i}$. Sendo que, $d_{i}$ é saída desejada do i-ésimo elemento da última camada da rede, $y_{i}$ é saída do i-ésimo elemento da última camada da rede e o $n s$ é o número do i-ésimo elemento da última camada da rede.

Tendo em consideração o neurônio de índice $i$ da rede e o método gradiente descendente, o ajuste do peso pode ser obtido pela equação(2) [7] [11] :

$$
V_{i}(h+1)=V_{i}(h)-\theta_{i}(h) .
$$

Sendo $\theta_{i}(h)=\gamma\left[\Delta_{i}(h)\right]$. Em que $\gamma$ é parâmetro de controle da estabilidade ou taxa de treinamento, $h$ representa o índice de iteração, $\Delta_{i}(h)$ é o gradiente do erro quadrático com relação aos pesos do neurônio $i$ avaliado em $h$ e $V_{i}$ é vetor contendo os pesos dos neurônios $i$.

Levando em consideração o problema apresentado, no qual corresponde à previsão de cargas elétricas, a função de ativação adotada foi a função de sigmoide. Uma função não-linear, definida pela equação (3)

$$
y_{i}=\frac{1}{1+\exp ^{-\lambda s_{i}}} .
$$

Sendo, $\lambda$ a constante que determina a inclinação da curva $y_{i}$ e $s_{i}=\sum_{i=1}^{n s}\left(X^{T} w\right)$.

À vista disso o treinamento do algoritmo de backpropagation, no qual seus pesos da rede são iniciados randomicamente no intervalo $(0,1)$, pode ser efetuado de duas maneiras, sendo elas: $\mathrm{O}$ primeiro procedimento fundamenta-se em ajustar os pesos da rede de todas as camadas, fazendo com que haja convergência para cada padrão, até que se complete o conjunto de entrada e esse processo é repetido até que ocorra a convergência da rede, ou seja, o erro quadrático seja inferior a uma tolerância preestabelecida para os padrões, já o outro procedimento é igual o anterior, porém se faz apenas uma iteração por padrão.

O algoritmo backpropagation é considerado um referencial em ternos de precisão, porém tem uma convergência bastante lenta. Diversas variações dos métodos têm sido proposto na literatura, com o objetivo de tornar esse processo de convergência mais eficiente. Entre eles tem-se o método de inserção de termo de momentum, o resilient-propagation e o Levenberg-Marquardt. 


\subsection{Algoritmo proposto}

Levando em consideração essas deficiências do backpropagation convencional, contendo alguns problemas que comprometem o seu desempenho, provocando lentidão no processo de convergência e não convergência. Tendo em vista essa problemática, este trabalho tem como propósito tornar esse processo de convergência mais eficiente com algoritmo backpropagation estocástico.

Um processo estocástico é determinado como uma coleção de variáveis aleatórias $X(t)$, a qual representa o estado do sistema no parâmetro, geralmente o tempo $t$. Deste modo, esse processo é frequentemente utilizado para estudar a evolução de fenômenos, ou sistemas, que são observados ao longo do sistema [4]. Diversos algoritmo de aprendizado já são baseados no método estocástico que seleciona probabilisticamente suas ações de ajustes, levando-se em conta um conjunto finito de possíveis situações, as quais são passíveis de se gerar resultados satisfatórios. Na literatura da área disseminam-se várias pesquisas focadas na utilização desse método estocástico no algoritmo backpropagation, porém não encontrado com objetivo de prever cargas elétricas.

O proposto previsor de cargas elétricas a curto prazo, por meio das redes neurais artificiais supervisionada, onde seu treinamento é através de combinações de padrões de entrada e saida, o vetor de entrada é extraído do banco de dados de uma companhia energética, e sua saída correspondente é calculada e comparada com a saída desejada. Empregando o algoritmo backpropagation estocástico, fundamentado no principio estatístico, considera-se que nem todos os pesos tem a mesma interferência no erro, onde sua atualização na rede acontece de forma randômica. Desenvolvendo-se uma diferente ferramenta para ajustar a lentidão e a não convergência do processo convencional, na tentativa de um melhor resultado.

\subsection{Aplicação e resultados}

Neste capítulo, serão abordados os principais resultados através do modelo proposto, via algoritmo backpropagation estocástico, visando resolver os problemas da previsão de carga a curto prazo (24 horas subsequentes). Dessa maneira, será utilizado o método de comparação entre: algoritmo backpropagation convencional e o backpropagation estocástico, com propósito de testar a eficiência da metodologia estocástica. Para a fase de treinamento da rede neural, serão empregados os dados históricos de uma companhia do setor elétrico brasileiro.

O sistema previsor neural estocástico foi programado na linguagem Fortran e o seu funcionamento resulta no campo estocástico de atualização do peso, de acordo com a porcentagem dada que nesse caso foi de 67 por cento dos pesos em cada iteração realizada de forma randômica.

As variáveis de entrada denotados neste trabalho, são os dados históricos de uma companhia do setor elétrico, do qual contêm valores de cargas horárias do ano de 1998, em que a primeira aplicação contem dados históricos de 21 dias (08/07/98 a 28/07/98), totalizando um conjunto de 504 vetores e tendo como objetivo prever o dia 29 de julho de 1998, e a segunda aplicação possui um conjunto com dados referentes a 42 dias (17/06/98 a 28/07/98, formando um total de 1008 vetores e visando prever o dia 29 de julho de 1998. De maneira geral, utiliza-se dimensão do vetor padrão de entrada 12 (dados dias da semana e horas codificadas na representação binária, mais carga horária e valores considerando-se três horas anteriores).

A análise dos resultados das duas redes neurais, sendo elas backpropagation convencional e a backpropagation estocástico, rede 1 e 2, respectivamente. Elaboradas com os mesmos parâmetros da rede (número de camadas, número de neurônios por camadas, tolerância, taxa de treinamento, momento e inclinação da função sigmóide), diferenciadas com apenas a aleatoriedade estocástica, que é feita de maneira randômica dos pesos a serem atualizados no decorrer da rede.

Na tabela 1 são apresentados os principais parâmetros referentes as redes neurais 1 e 2 utilizadas e seu treinamento: backpropagation convencional (BP) e o backpropagation estocástico (BPesto), respectivamente. 
Tabela 1: Especificação da rede neural referente à aplicação 1 e 2

\begin{tabular}{ccc}
\hline Item & Valor (Aplicações 1 e 2) & \\
\hline & Rede Neural 1 (BP) & Rede Neural 2 (BPesto) \\
\hline Número de vetores & $504 / 1008$ & $504 / 1008$ \\
Número de camadas & 3 & 3 \\
Número de neurônios por camada & $12-30-1$ & $12-30-1$ \\
Tolerância & 0,05 & 0,05 \\
Taxa de Treinamento $\gamma *$ & $4,5 / 5,5$ & $4,5 / 5,5$ \\
Termo momento $\eta$ & 0,9 & 0,9 \\
Inclinação da função sigmóide $\lambda$ & 0,3 & 0,3 \\
Taxa de porcentagem atualização dos pesos & $100 \%$ & $67 \%$ \\
\hline
\end{tabular}

Para uma análise comparativa, define-se o erro porcentual absoluto médio, MAPE apresentado na equação (4) e o erro máximo da previsão diária, denotado na equação (5), comparando os valores reais da carga com os valores estimados através das redes neurais da seguinte maneira:

$$
\begin{gathered}
\text { MAPE }=\frac{1}{N}\left\{\sum_{i=1}^{N} \frac{(|L(h)-L E(h)|)}{L(h)}\right\} \times 100 . \\
\operatorname{Erro}_{m} \operatorname{aximo}(\%)=\max \left\{\frac{(|L(h)-L E(h)|)}{L(h)}\right\} \times 100 .
\end{gathered}
$$

Sendo, $L(h)$ o valor da carga real referente a hora $h$ e a variável $L E(h)$ é o valor da carga estimada pela rede neural referente à hora $h$. Tendo em vista que o número total de horas $(24$ horas), tem como referencia o termo $N$.

Na figura 1 , são apresentados os resultados da previsão de carga desses treinamentos, das aplicações 1 e 2, referente ao dia 29 de julho de 1998:
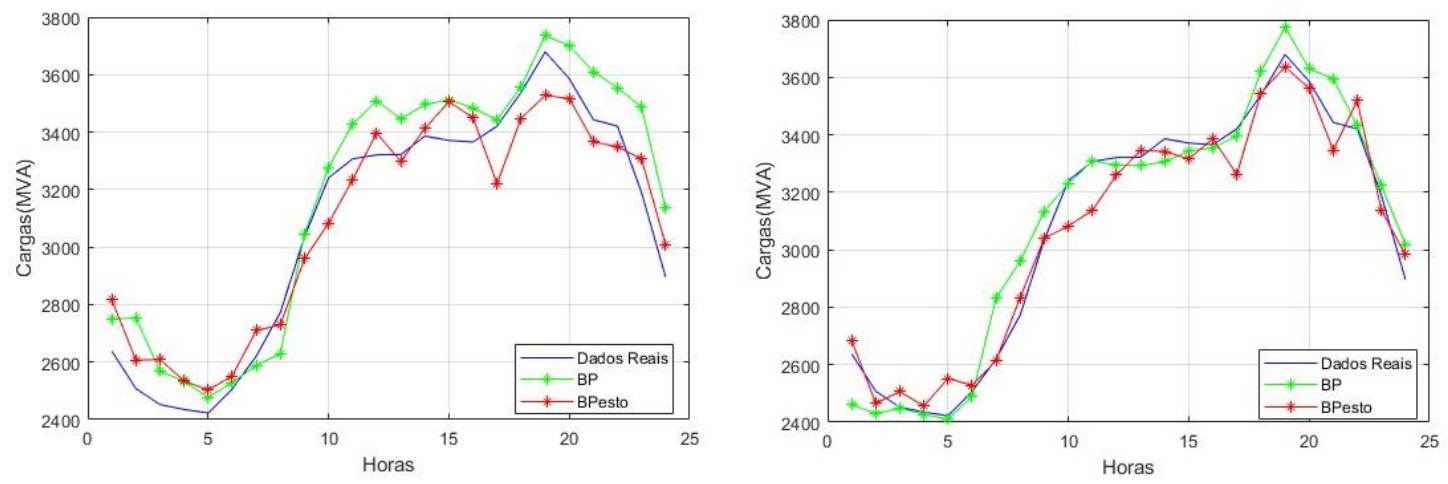

Figura 1: Resultado da Previsão de Cargas para Rede Neural 1 (BP) e 2 (BPesto), aplicações 1 e 2.

Os resultados da previsão de cargas denotados na figura 1, para redes neurais com os treinamentos backpropagation convencional (BP) e o backpropagation estocástico (BPesto), e as aplicações 1 com 504 vetores e aplicação 2 com 1008 vetores, são apresentados na tabela 2, e aplicados como métodos comparativos em termos de número de ciclos, tempo de processamento, erro médio e o erro máximo. 
É importante ressaltar que a taxa de porcentagem da atualização de pesos operadas na aplicação 1 e 2 no treinamento backpropagation estocástico, teve um processo computacional por depuração até que chegasse em um melhor resultado. Nota-se que a as diferenças dessas aplicações são apontadas na tabela 1. Observa-se que os erros gerados pelo método proposto no trabalho, o do backpropagation estocástico é menor comparado aos resultados do backpropagation convencional, dessa maneira torna-se mais eficiente para um sistema previsor de cargas elétricas.

Demostra-se assim o principio estatístico, do qual não é necessário atualização de todos os pesos da rede e levando em consideração que nem todos tem a mesma influência no erro, pode-se então atualizar e escolher os pesos randomicamente, sem nenhuma penalidade na complexidade da implementação e com resultados positivos, e melhor convergência.

Tabela 2: Resultados comparativos para as aplicações 1 e 2

\begin{tabular}{ccc}
\hline Item & BP Convencional & BP Estocástico \\
\hline & Rede Neural 1 & Rede Neural 2 \\
\hline Número ciclos & $162 / 171$ & $1068 / 1768$ \\
Tempo de processamento (s) & $1,76 / 2,11$ & $95 / 240$ \\
Erro médio(\%) & $3,75 / 2,16$ & $3,22 / 2$ \\
Erro máximo(\%) & $9,8 / 8,1$ & $6,73 / 5,35$ \\
\hline
\end{tabular}

\section{Conclusões}

O previsor de cargas elétricas desenvolvido, neste trabalho e seus resultados obtidos são de suma importância no contexto da literatura, tendo potencial para aplicações em problemas complexos, com parâmetros diferentes e número maiores de variáveis e informações. Foram analisados e executados vários testes, com base de dados históricos de uma companhia do setor elétrico brasileiro.

Por meio dessa análise de resultados obtidos, serviram de parâmetros para verificar a eficiência do algoritmo backpropagation estocástico e o que poderia ser melhorado. Dessa maneira, por processo de depuração, foi encontrada uma porcentagem de atualização de pesos, que proporcionou melhores e mais eficientes resultados do que o algoritmo convencional.

Os resultados de previsão de carga a curto prazo, considerando as 24 horas subsequentes, obtidos pelo método proposto, mostraram uma leve melhora em relação aos resultados obtidos pelo algoritmo backpropagation convencional. Uma observação importante neste sentido, uma vez que, existe uma melhora no treinamento estocástico no qual mostra que não são necessários a adaptação de todos os neurônios da rede, mas apenas alguns que foram selecionados aleatoriamente. Desta forma, tem-se que associando o procedimento estocástico com a identificação dos neurônios que devem ser atualizados possa gerar um modelo mais robustos que obtenha bons resultados. Podendo concluir, que o algoritmo backpropagation estocástico tornou-se mais eficiente para um sistema previsor de cargas elétricas.

Como perspectivas futuras sugere-se o estudo e o aperfeiçoamento dos resultados obtidos, acrescentando uma sub rotina na programação do algoritmo para encontrar de maneira estocástica o melhor balanceamento de atualização de pesos.

\section{Agradecimentos}

O presente trabalho foi realizado com o apoio financeiro do Conselho Nacional de Desenvolvimento Científico e Tecnológico (CNPq) e da Coordenação de Aperfeiçoamento de Pessoal de Nível 
Superior - Brasil (CAPES) - Código de Financiamento 001.

\section{Referências}

[1] Guirelli, C. R. Previsão da carga de curto prazo de áreas elétricas através de técnicas de inteligência artificial, Tese (Doutorado) - Universidade de São Paulo, 2006.

[2] Haykin. A comprehensive foundation, Publications Neural networks, volume 2, chapter 2004, pages 41,2004 .

[3] Hodgkin, A. L. and Huxley, A. F. A quantitative description of membrane current and its application to conduction and excitation in nerve, The Journal of physiology, Wiley-Blackwell, v.117, n.4, p.500, 1952.

[4] Nogueira, F. Cadeias de Markov, Notas de Aula, Universidade Federal de Juiz de Fora, 2009.

[5] O'Donovan, T. M. Short term forecasting: An introduction to the Box-Jenkins approach, JOHN WILEY \& SONS, INC., 605 THIRD AVE., NEW YORK, NY 10158, USA, 1983, $256,1983$.

[6] Silva, I. D., Spatti, D. H. e Flauzino, R. A. Redes neurais artificiais para engenharia e ciências aplicadas, Sage São Paulo: Artliber, v.23, n.5, p.33-111, 2010.

[7] Simpson, P. K. Artificial neural systems: foundations, paradigms, applications, and implementations, Elsevier Science Inc., 1989.

[8] Tang, Z., Almeida, C. D. and Fishwick, P. A. ITime series forecasting using neural networks vs. Box-Jenkins methodology, Sage Publications Sage CA: Thousand Oaks, CA, v.57, n.5, p. 303-310, 1991.

[9] Wasserman, P. D. Advanced methods in neural computing. Publications,John Wiley \& Sons, Inc., 1993.

[10] Werbos, P. J. The roots of backpropagation: from ordered derivatives to neural networks and political forecasting, John Wiley \& Sons, v.1, 1994.

[11] Widrow, B. and Lehr, M. A. 30 years of adaptive neural networks: perceptron, madaline, and backpropagation, Proceedings of the IEEE, IEEE, v.78, n.9, p. 1415-1442, 1994. 